\title{
Signet Ring Cell Adenoma of the Thyroid: A Very Rare Entity
}

\author{
Tulin Yalta ${ }^{a}$ Sahende Elagoz ${ }^{c}$ Meral Uyar ${ }^{a}$ Omer Topuz $^{b}$ Hatice Ozer \\ Ersin Tuncer ${ }^{c}$ \\ Departments of a Pathology and ${ }^{b}$ General Surgery, Sivas State Hospital, and ${ }^{\mathrm{c} D e p a r t m e n t}$ of Pathology, \\ Cumhuriyet University, Sivas, Turkey
}

\section{Key Words}

Signet ring cell adenoma $\cdot$ Thyroid lesion $\cdot$ Metastatic signet ring cell carcinoma

\begin{abstract}
Objectives: To report a case of signet ring cell adenoma of the thyroid which is an extremely rare thyroid lesion. Clinical Presentation and Intervention: A 25-year-old female patient presented with a goiter and dysphagia that had been present for the last 1 year before admission. Physical examination revealed a palpable solitary nodule in the right lobe of the thyroid. The ultrasonogram demonstrated multiple nodules among which the most remarkable one was $15 \times$ $24 \mathrm{~mm}$ in size, in the right lobe of the thyroid. After surgical excision, the lesion was found to be consistent with signet ring cell adenoma characterized by the presence of round to oval signet ring cells with large cytoplasmic vacuoles and hyperchromatic eccentric nuclei. Intracytoplasmic thyroglobulin, periodic acid-Schiff (PAS) with and without diastase and combined Alcian-blue-PAS were all positive. Conclusions: Pathologists should keep this rare primary tumor of the thyroid in mind when examining thyroid lesions and should not confound it with metastatic signet ring cell carcinoma of the thyroid.

Copyright $\odot 2009$ S. Karger AG, Basel
\end{abstract}

\section{Introduction}

Squamous, oncocytic or clear cell (with signet ring formation) metaplastic changes may take place in the thyroid tissue [1]. Among these, clear cell (with signet ring formation) is the most unusual variant, and adenoma of the thyroid with signet ring formation has been very rarely encountered [1]. Signet ring cell adenoma of the thyroid usually manifests as a palpable and solitary neck mass. Due to the slight symptomatology, the admission of patients may be delayed. Physical examination generally does not confer any diagnostic clue to discriminate it from other neck masses, and absolute diagnosis is based on pathological examination. The patient described below also had a neck mass with slight symptomatology, and was found to suffer this unusual thyroid lesion.

\section{Case Report}

A 25-year-old female patient presented with a goiter and dysphagia that had been present for the last 1 year before admission. Physical examination revealed a palpable solitary nodule in the right lobe of the thyroid. The other clinical and laboratory examinations, including thyroid hormone levels, were unremarkable. The ultrasonogram demonstrated multiple nodules among which the most remarkable one was $15 \times 24 \mathrm{~mm}$ in size, in the right lobe of the thyroid. A right hemithyroidectomy was carried

Dr. Tulin Yalta

Department of Pathology, Sivas State Hospital

TR-58100 Sivas (Turkey)

Tel. +90 505396 7908, Fax +90 3462408 413, E-Mail tdeyalta@gmail.com 


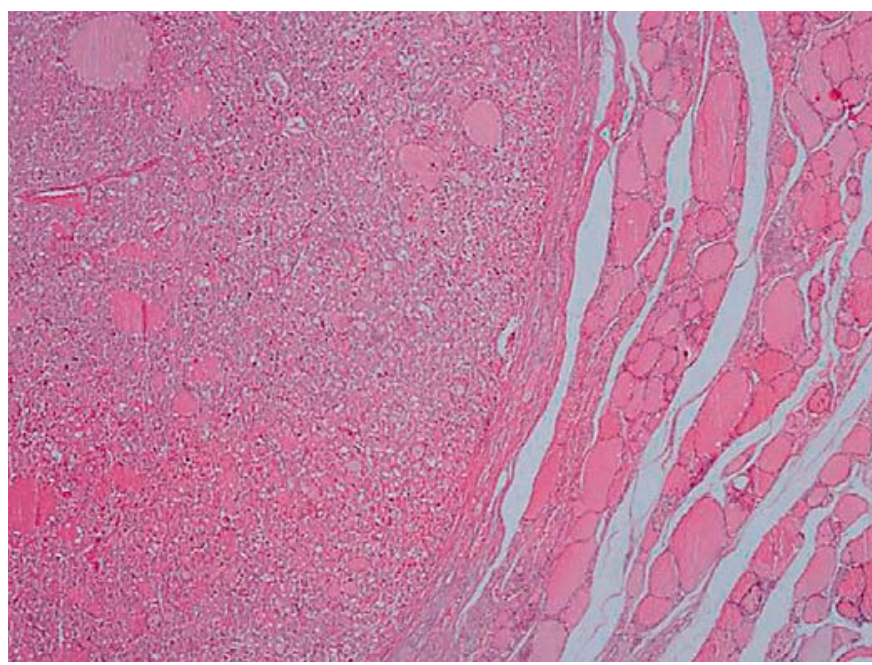

Fig. 1. Microscopy of the signet ring cell adenoma enclosed within a fibrous capsule. HE. $\times 40$.

out. On macroscopy, the right lobe of the thyroid was found to contain a well-circumscribed, elastic nodule $(20 \times 15 \times 5 \mathrm{~mm}$ in size) with foci of hemorrhage. There was no local infiltration of the tumor. Histologically, the nodule was found to contain signet ring cells that were round to oval in shape. Signet ring cells had large cytoplasmic vacuoles and hyperchromatic eccentric nuclei. There was also an intact capsule surrounding the lesion. The microscopy of the tumor was found to be consistent with signet ring cell adenoma. In immunocytochemistry, intracytoplasmic thyroglobulin was positive and calcitonin was negative. In mucin histochemistry, periodic acid-Schiff (PAS) with and without diastase and Alcian blue-PAS were both positive for intracytoplasmic mucin. The photomicrographs are shown in figures 1 and 2. After an uneventful recovery, the patient was discharged with the recommendation of close follow-up.

\section{Discussion}

Histopathological studies on signet ring cell adenoma of the thyroid have been limited due to the scarcity of this tumor compared to other thyroid lesions. The mechanisms underlying the formation of signet ring cells are still obscure. Only a few authors have proposed theories to explain this issue. Signet ring cells have been known to contain immunoreactive thyroglobulin and generally stain positively for mucin [2], suggesting that intracytoplasmic accumulation of thyroglobulin contributes solely to the signet ring formation [2]. In the present case, signet ring cells stained positively with PAS with and without diastase and combined Alcian blue-PAS. Mochizuki et al. [3] proposed that dysfunctional dilated rough endoplas-

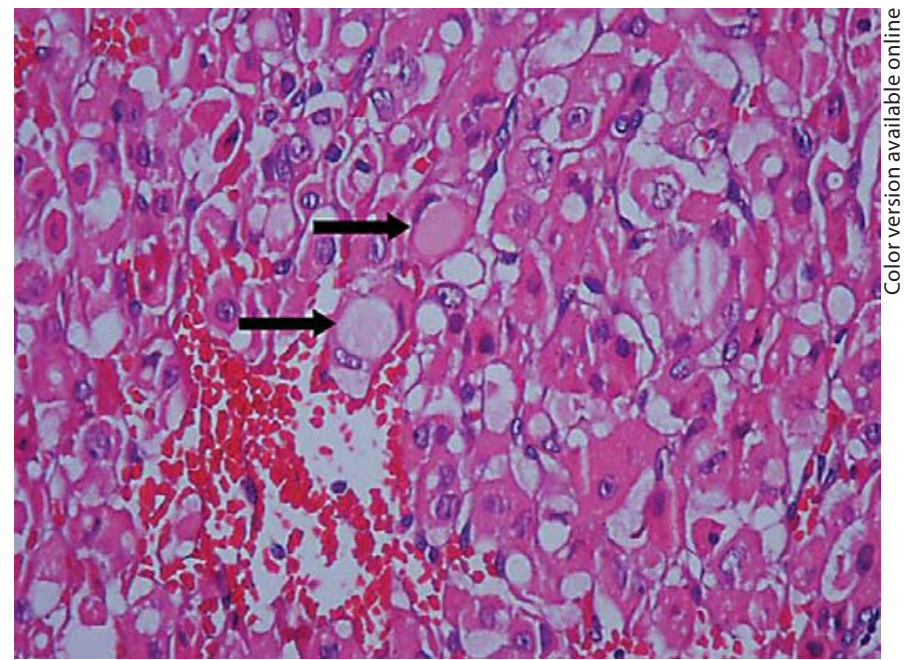

Fig. 2. Microscopy demonstrating typical signet ring cells (black arrows) with foci of hemorrhage. HE. $\times 400$.

mic reticulum (compressing the nucleus to the apical portion) or Golgi apparatus might contribute to the signet ring cell formation.

Signet ring cell adenoma of the thyroid has been reported very rarely in the literature. El-Sahrigy et al. [1] reported a 48-year-old female with signet ring follicular adenoma of the thyroid. They emphasized that unfamiliarity with this tumor might lead to a misdiagnosis of metastatic signet ring cell carcinoma. Mochizuki et al. [3] reported a 46-year-old Japanese euthyroid woman with this tumor, while Yoshida et al. [4] reported a 22-year-old male patient with signet ring cell adenoma of the thyroid which had poor staining for thyroglobulin despite the predominance of mucin. Rigaud et al. [5] reported a case of microfollicular thyroid adenoma with mucin secretion that was uniformly composed of sulfated acid mucins. Gulwani and Chopra [6] reported the first case with this thyroid tumor in the Indian literature. The authors emphasized that exclusion of the primary signet ring cell carcinoma in another site of the body is essential before diagnosing the thyroid mass as a signet ring cell adenoma [6]. They also stated that immunohistochemical staining for thyroglobulin is a useful marker in discriminating signet ring cell adenomas from metastatic signet ring cell carcinomas [6]. Murakami et al. [7] reported an unusual case of thyroid adenoma with extensive mucin deposition in the extracellular space. This unusual finding is also termed extensive myxoid stroma, and is thought to be associated with differentiation of thyroid follicular cells into mucin-producing cells [7]. 
Patients with neck masses may present with symptoms including dysphagia, dyspnea, and chronic neck pain. The majority of patients with this tumor are asymptomatic or slightly symptomatic due to its noninvasive nature. The present case had suffered mild dysphagia. This symptom might be considered as coincidental, rather than being a tumor-associated symptom, since the tumor was found to be small in size and noninfiltrative in nature.

\section{Conclusions}

The present case is one of the very rarely reported cases of signet cell adenoma of the thyroid. Pathologists should be aware of this rare primary tumor of the thyroid, and should not confound it with other thyroid lesions, including metastatic signet ring cell carcinoma of the thyroid.

\section{References}

1 El-Sahrigy D, Zhang XM, Elhosseiny A, Melamed MR: Signet-ring follicular adenoma of the thyroid diagnosed by fine needle aspiration. Report of a case with cytologic description. Acta Cytol 2004;48:87-90.

$\checkmark 2$ Gherardi G: Signet-ring cell 'mucinous' thyroid adenoma: a follicle cell tumour with abnormal accumulation of thyroglobulin and a peculiar histochemical profile. Histopathology 1987;11:317-326.
3 Mochizuki M, Saito K, Kanazawa K: Benign follicular thyroid nodule composed of signet-ring like cells with PAS-negative thyroglobulin accumulation in dilated rough endoplasmic reticulums. Acta Pathol Jpn 1992; 42:111-114.

4 Yoshida J, Tanimura A, Yamashita H, Matsuo K: Signet-ring cell adenoma of the thyroid with mucin predominance. Thyroid 1999;9:401-404.
5 Rigaud C, Peltier F, Bogomoletz WV: Mucin producing microfollicular adenoma of the thyroid. J Clin Pathol 1985;38:277-280.

6 Gulwani H, Chopra P: Mucin-producing signet ring cell adenoma of the thyroid. Indian J Pathol Microbiol 2008;51:507-508.

7 Murakami S, Sakata H, Okubo K, Tsuji Y, Kayano H: Thyroid adenoma with extensive extracellular mucin deposition: report of a case. Surg Today 2007;37:226-229. 